\title{
Low-temperature time-resolved cathodoluminescence study of exciton dynamics involving basal stacking faults in a-plane GaN
}

\author{
P. Corfdir, ${ }^{1, a)}$ J. Ristić, ${ }^{1}$ P. Lefebvre, ${ }^{1,2}$ T. Zhu, ${ }^{1}$ D. Martin, ${ }^{1}$ A. Dussaigne, ${ }^{1}$ J. D. Ganière, ${ }^{1}$ \\ N. Grandjean, ${ }^{1}$ and B. Deveaud-Plédran ${ }^{1}$ \\ ${ }^{1}$ Institut de Photonique et d'Electronique Quantiques, Ecole Polytechnique Fédérale de Lausanne (EPFL), \\ 1015 Lausanne, Switzerland \\ ${ }^{2}$ Groupe d'Etude des Semiconducteurs, CNRS-Université de Montpellier II, 34095 Montpellier, France
}

(Received 17 March 2009; accepted 4 May 2009; published online 22 May 2009)

\begin{abstract}
Time-resolved cathodoluminescence at $27 \mathrm{~K}$ has been performed on $a$-plane $\mathrm{GaN}$ grown by epitaxial lateral overgrowth. We detail the relaxation and recombination mechanisms of excitons [free or bound to neutral donors, or bound to $I_{1}$-type basal stacking faults (BSFs)] in relation to the local density in BSFs. We describe the slow exciton capture rate on isolated BSFs by a diffusion model involving donors via a hopping process. Where BSFs are organized into bundles, we relate the shorter rise time to intra-BSF localization processes and the multiexponential decay to the type-II band alignment of BSFs in wurtzite GaN. (C) 2009 American Institute of Physics.
\end{abstract}

[DOI: $10.1063 / 1.3142396]$

Wurtzite group-III nitrides are nowadays the materials of choice for optoelectronic devices emitting visible-UV light. ${ }^{1}$ However, the presence in heterostructures of huge built-in electric fields along the $c$-axis ${ }^{2}$ decreases the overlap between the electron and hole wave functions and therefore increases the influence of nonradiative processes at room temperature. ${ }^{3}$ The growth of nitride heterostructures along the $a$-axis-a nonpolar direction perpendicular to the $c$-axis-is a way to overcome the quantum-confined Stark effect ${ }^{4}$ and to enhance the radiative efficiency of the devices. However, the growth of high-quality $a$-plane $\mathrm{GaN}$ is complex, and still gives rise to large densities of extended defects. $^{5-7}$ In particular, basal stacking faults (BSFs) of different kinds constitute extended defects with optical activity, which have been studied both theoretically ${ }^{8,9}$ and experimentally. ${ }^{10-12}$ They are generally considered as narrow (a few atomic monolayers) type-II quantum wells made of cubic-like GaN surrounded by wurtzite $\mathrm{GaN}$ barriers. Recently, cathodoluminescence (CL) studies of epitaxial lateral overgrowth (ELO) $a$-plane GaN showed that $I_{1}$-type BSFs tend to gather into dense bundles, evidencing positiondependent morphologies. ${ }^{12}$ In the high-quality, fast-grown " $+c$ wings," those bundles give rise to intense CL stripes at
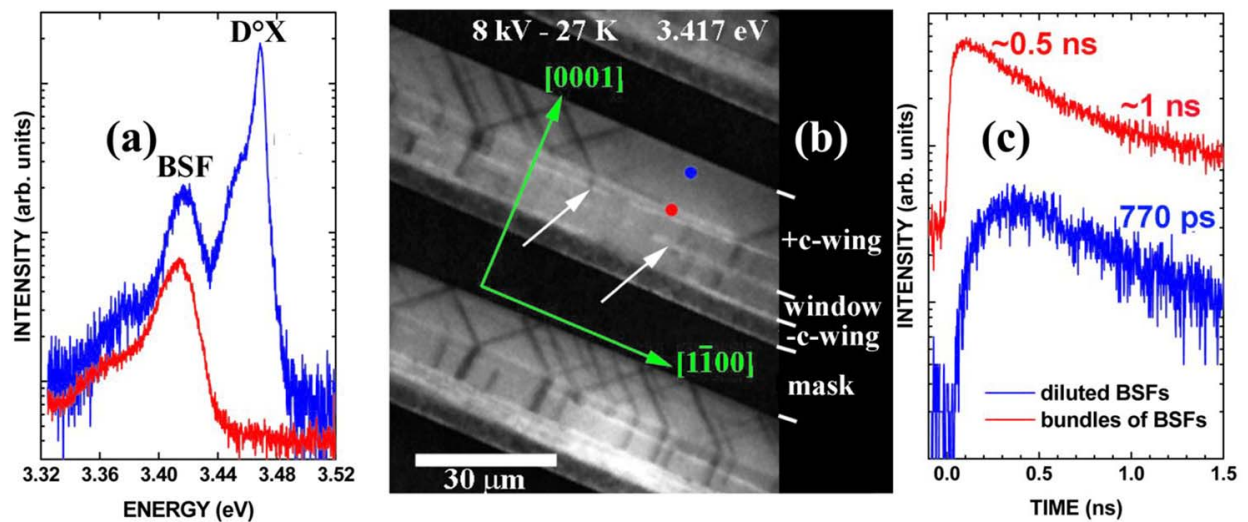

FIG. 1. (Color online) Blue (respectively red) curves correspond to excitation away from (respectively on) bundles of BSFs, as shown in (b) by a blue (respectively red) dot. (a) Timeintegrated CL spectra (upper curve is blue). (b) Top-view CL map taken at $3.417 \mathrm{eV}$. White arrows point out the emergence of bundles of BSFs. (c) Time decays of the $3.417 \mathrm{eV}$ CL signal (upper curve is red).

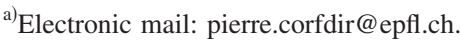


setup of a cryogenic system based on liquid-helium cooling of a copper sample holder now allows us to routinely reach temperatures as low as $27 \mathrm{~K}$.

In this letter, we therefore use low-temperature pTRCL to obtain quantitative information on exciton diffusion and recombination in an ELO $a$-plane GaN sample. We clearly confirm that, depending on the region of the sample, BSFs are either diluted or concentrated and, for different excitation spots, we thoroughly measure the CL rise and decay times for all excitonic lines. We propose a diffusion model that emphasizes the role of donors in the dynamics of exciton trapping by BSFs.

Our $22 \mu \mathrm{m}$ thick $a$-plane GaN epilayer has been grown by hydride vapor phase epitaxy using the ELO technique (ELO wings width comprised between 20 and $30 \mu \mathrm{m}$ ). ${ }^{5,6}$ We purposely stopped the growth before the coalescence of the ELO wings in order to study separately the $+c$ and $-c$ wings. Our pTRCL setup uses a picosecond pulsed electron gun that focuses electrons onto the sample surface with a 50 $\mathrm{nm}$ accuracy. We used an acceleration voltage of $8 \mathrm{kV}$ and obtained a probe current comprised between 1 and $100 \mathrm{pA}$ at the sample surface. Electron-hole pairs are generated within a pear-shaped volume, which in-plane extension is well described by a two-dimensional Gaussian distribution. ${ }^{16}$ The luminescence of the samples was analyzed by a 600 grooves per $\mathrm{mm}$ grating followed by a streak camera synchronized with the pulsed electron gun, yielding temporal resolution of 10 ps.

Figure 1(a) shows time-integrated CL spectra of our sample. Based on previous studies, ${ }^{11}$ we assign the emission lines at 3.471 and $3.417 \mathrm{eV}$ to donor-bound exciton $\mathrm{A}\left(\mathrm{D}^{\circ} \mathrm{X}\right)$ and to BSF-bound exciton, respectively. On the high energy side of the $\mathrm{D}^{\circ} \mathrm{X}$ lies the emission from free exciton $\mathrm{A}(\mathrm{FX})$ at $3.478 \mathrm{eV}$, to be taken into account in a careful deconvolution procedure. Figure 1(b) shows top-view time-integrated CL map of our ELO GaN epilayer at $T=27 \mathrm{~K}$, taken at the luminescence energy of $I_{1}$-BSFs. Despite the higher density in BSF in the $-c$ wings, one can note that the BSF emission in these regions is weak, to be related with high local densities of nonradiative defects that dominate the recombination mechanisms. On this image, we also distinguish in the $+c$ wings the bright bundles and the diluted zones. In the diluted zones, the CL spectrum and dynamics [Figs. 1(a) and 1(c)] remain absolutely unchanged when we change, from a few hundred nanometers to several micrometers, the distance between the excitation spot and the nearest bundle. In particular, the rise-times of the donor- and BSF-bound excitons CL do not depend on this distance, confirming that BSFs are homogeneously present in those regions.

In those regions, we measured the $\mathrm{CL}$ decay dynamics of free-, donor-, and BSF-bound excitons (Fig. 2). The FX line decays exponentially with a time constant of $85 \mathrm{ps}$, which corresponds to the rise-time of the $\mathrm{D}^{\circ} \mathrm{X}$ luminescence. The mechanism limiting the FX lifetime is therefore their capture by donors. The $\mathrm{D}^{\circ} \mathrm{X}$ line decays exponentially with a time constant of 135 ps. More surprising, the BSF CL, which decays exponentially with a time constant of $770 \mathrm{ps}$, exhibits a very slow rising-the maximum intensity is reached $\sim 300$ ps after the excitation. A priori, one would expect the BSFs either to be directly populated by the excitation pulse or to capture free excitons by a scattering process. In both cases, given the measured decay time for free excitons, the

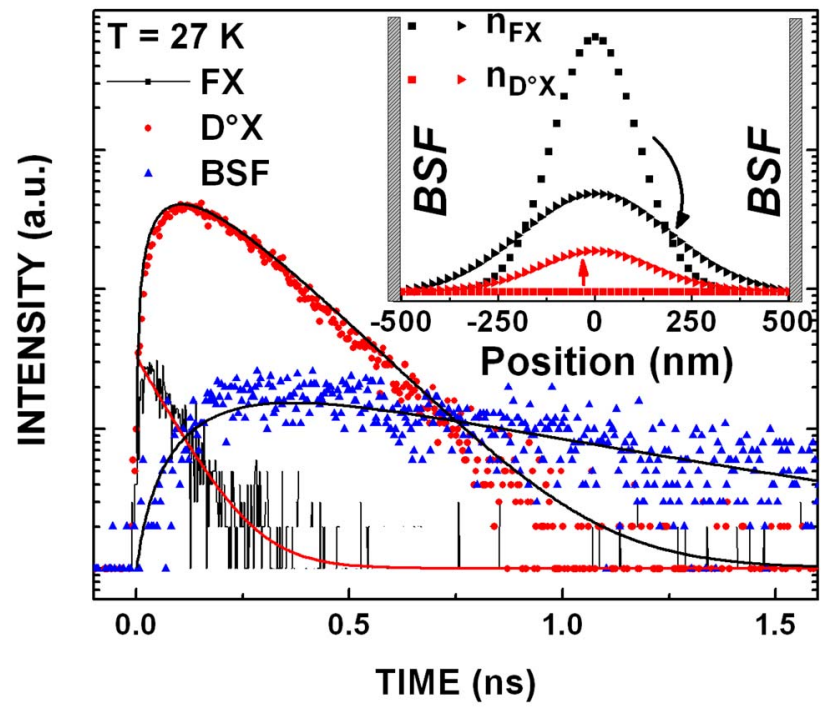

FIG. 2. (Color online) Luminescence decays of FX, ${ }^{\circ} \mathrm{X}$, and BSF excitons when excitation is on "diluted" regions. Solid lines are the result of the diffusion model. The latter is sketched in the insert showing the calculated spatial distributions of $\mathrm{FX}$ and $\mathrm{D}^{\circ} \mathrm{X}$ (black and red symbols, respectively) at $t=0$ and $t=50$ ps (squares and triangles).

BSF rise-time should not exceed a few tens of picoseconds, which is obviously not what we observe. The mechanism by which the BSFs trap excitons is therefore more complex, although it necessarily involves the diffusion of excitons.

We have therefore developed a one-dimensional diffusion model for excitons along the $c$-axis, i.e., perpendicular to the BSF planes. As sketched in the insert of Fig. 2, we assumed two BSFs separated by typically $1 \mu \mathrm{m}$, consistently with transmission electron microscopy that revealed, in the $+c$ wings of the ELO epilayer, typical BSF densities of $10^{4} \mathrm{~cm}^{-1}{ }^{13}$ Using the model developed in Ref. 16 and derived for $\mathrm{GaN},{ }^{17}$ we account for the pulsed electron-beam excitation at $t=0$ by a Gaussian distribution of free excitons, with half-width of $\sim 200 \mathrm{~nm}$, along the $c$-axis, centered between the two BSFs. These free excitons then diffuse with the ambipolar diffusion coefficient $D_{\mathrm{FX}}=4 \mathrm{~cm}^{2} \mathrm{~s}^{-1}$ determined independently ${ }^{18}$ and can also recombine radiatively and nonradiatively (lifetimes $\tau_{\mathrm{R}}$ and $\tau_{\mathrm{NR}}$ ), or be captured by neutral donors $\left(\tau_{C}\right)$ or BSFs. For the latter process, as soon as a free exciton reaches a BSF, we consider it instantaneously captured. BSF-bound excitons can only recombine radiatively with decay time $\tau_{\mathrm{BSF}}$, whereas the radiative lifetime for donor-bound excitons is $\tau_{\mathrm{D}^{\circ} \mathrm{X}}$. A crucial ingredient of the model is the time $\tau_{\mathrm{ESC}}$, characteristic of the thermally assisted escape of excitons from neutral donors.

The insert in Fig. 2 illustrates the modeled timedependence of the $\mathrm{FX}$ and $\mathrm{D}^{\circ} \mathrm{X}$ density profiles. The parameters are those that allowed us to fit simultaneously the relative intensities, rising and decaying dynamics of all three types of excitons (Fig. 2) for an inter-BSF distance of $1 \mu \mathrm{m}$. Given the fast capture of excitons onto donors, and despite some diffusion of those $\mathrm{FX}$ toward the $\mathrm{BSFs}$, the $\mathrm{D}^{\circ} \mathrm{X}$ distribution after a few tens of picoseconds is quite similar to the initial distribution of FX. Therefore, if we do not include the thermally assisted reemission of excitons from the donors, we cannot fit the observed long rise-time for the BSF signal because the BSFs would receive a very small amount of excitons with a short time constant. 
The best fit to our experimental results yields $\tau_{\text {ESC }}$ $=600 \mathrm{ps}, \tau_{C}=150 \mathrm{ps}, \tau_{\mathrm{D}^{\circ} \mathrm{X}}=140 \mathrm{ps}$, and $\tau_{\mathrm{BSF}}=770 \mathrm{ps}$. The latter two values are close to the measured CL decay times and they are therefore determined with an excellent accuracy. The same comment goes to the capture time of FX onto donors $\left(\tau_{C}\right)$, which controls both the decay rate of FX, and the rise rate of the $\mathrm{D}^{\circ} \mathrm{X}$ transition. We also find $\tau_{\mathrm{NR}}$ $=600 \mathrm{ps}$ and $\tau_{\mathrm{R}}=6000 \mathrm{ps}$, but these values are estimated with a lower accuracy. The FX radiative and nonradiative recombinations are not the mechanisms that limit the FX lifetime, but rather control the instantaneous intensity.

Concerning the role played by donors in the diffusion process, we must stress the fact that the escape time $\tau_{\mathrm{ESC}}$ is neither independent on the inter-BSF distance, nor on the fixed diffusion coefficient $D_{\mathrm{FX}}$. Nevertheless, whatever the values that we tried to set for $D_{\mathrm{FX}}$ or for the inter-BSF distance, we absolutely need to include the thermally assisted escape of excitons from donors in order to fit the fast decay of FX, and both the fast and slow rise-times of $\mathrm{D}^{\circ} \mathrm{X}$ and BSFs CL. This demonstrates that, for $T=27 \mathrm{~K}$, the excitons that are created in the diluted regions diffuse toward the BSFs via the donors, by a kind of hopping process. Temperature-dependent pTRCL experiments would usefully complement these results, but we cannot yet ensure the mechanical stability of our nanodisplacements at intermediate temperatures to perform such experiments.

Our model is not meant to describe the case of direct excitation of bundles, where BSFs are typically separated from each other by $10 \mathrm{~nm}$, i.e., only three times the donor (or exciton) Bohr radius, and smaller by a factor of 1.4 than the characteristic diameter of the $\mathrm{D}^{\circ} \mathrm{X}$ complex. ${ }^{19}$ Consequently, (i) the electron beam excites simultaneously several BSFs, and (ii) the lifetimes of free- and donor-bound excitons should be dramatically short. This is confirmed by the experimental results shown in Fig. 1(a). Only the BSF-related line at $3.417 \mathrm{eV}$ is observed. Its rise-time is much shorter than the one observed in the diluted regions [Fig. 1(c)], but the maximum intensity is reached after $\sim 110 \mathrm{ps}$, which means that the population of BSF excitons is not established instantaneously. We tentatively relate this observation to slow relaxation mechanisms of BSF-bound excitons toward deep potential fluctuations inside BSFs themselves. These intra-BSF localization processes have already been reported, ${ }^{12,20}$ although not yet thoroughly interpreted.

The decay dynamics of the BSF-related CL are clearly different when excitons are created on (multiexponential decay) and away from (exponential) the bundles [Fig. 1(c)]. We observe on the bundles components faster $(0.5 \mathrm{~ns})$ and slower (1 ns) than the decay of diluted BSFs. This suggests that, in the bundles, the overlap of electron and hole wave functions presents a large variety that we relate to the distribution of inter-BSF distances. This variety may be an indirect proof of the type-II band alignment for the BSFs, the large presence probability of the hole in the wurtzite GaN "barriers" yielding a diversity of inter-BSF coupling schemes. In case of type-I, electron and hole wave functions are both pinned on the BSF plane, what should lead to a variety in lifetime with lower amplitude. However, detailed excitonic calculations are needed to confirm this hypothesis, and to explain both the faster and the slower lifetimes in the bundles.

In summary, we have used the low-temperature pTRCL technique to investigate in detail the mechanisms of exciton relaxation and recombination involving BSFs in an $a$-plane GaN epilayer grown by the ELO method. Our results confirm that the BSFs exhibit two types of spatial distributions. In the regions where they are diluted, the relaxation of excitons toward them, for $T=27 \mathrm{~K}$, involves the donors via a kind of hopping process. In the regions where they are concentrated into bundles, the exciton relaxation is much faster but not instantaneous, which we tentatively ascribe to intraBSF localization processes. The decay dynamics of the BSF CL was also found different, depending on whether the excitation region has a small or high density of BSFs, which may indirectly confirm the type-II band alignment expected for $I_{1}$-BSFs in GaN.

The authors wish to thank the Attolight Co. for the constant improvement of the pTRCL set-up, R. Rochat, N. Leiser, Y. Trolliet and D. Trolliet for technical assistance. We acknowledge financing by Swiss National Science Foundation through Quantum Photonics NCCR and Project No. 119840.

${ }^{1}$ S. Nakamura, T. Mukai, and A. Senoh, Appl. Phys. Lett. 64, 1687 (1994). ${ }^{2}$ P. Lefebvre, A. Morel, M. Gallart, T. Taliercio, J. Allègre, B. Gil, H. Mathieu, B. Damilano, N. Grandjean, and J. Massies, Appl. Phys. Lett. 78, 1252 (2001).

${ }^{3}$ P. Lefebvre, T. Taliercio, A. Morel, J. Allègre, M. Gallart, B. Gil, H. Mathieu, B. Damilano, N. Grandjean, and J. Massies, Appl. Phys. Lett. 78, 1538 (2001).

${ }^{4}$ N. Akopian, G. Bahir, D. Gershoni, M. D. Craven, J. S. Speck, and S. P. DenBaars, Appl. Phys. Lett. 86, 202104 (2005).

${ }^{5}$ C. F. Johnston, M. J. Kappers, J. S. Barnard, and C. J. Humphreys, Phys. Status Solidi C 5, 1786 (2008).

${ }^{6}$ X. Ni, Ü. Özgür, Y. Fu, N. Biyikli, J. Xie, A. A. Baski, H. Morkoç, and Z. Liliental-Weber, Appl. Phys. Lett. 89, 262105 (2006).

${ }^{7}$ B. Imer, F. Wu, S. P. DenBaars, and J. S. Speck, Appl. Phys. Lett. 88, 061908 (2006).

${ }^{8}$ C. Stampfl and C. G. Van de Walle, Phys. Rev. B 57, R15052 (1998).

${ }^{9}$ Y. T. Rebane, Y. G. Shreter, and M. Albrecht, Phys. Status Solidi A 164, 141 (1997).

${ }^{10}$ G. Salviati, M. Albrecht, C. Zanotti-Fregonara, N. Armani, M. Mayer, Y Shreter, M. Guzzi, Y. V. Melnik, K. Vassilevski, V. A. Dmitiev, and H. P. Strunk, Phys. Status Solidi A 171, 325 (1999).

${ }^{11}$ R. Liu, A. Bell, F. A. Ponce, C. Q. Chen, J. W. Yang, and M. A. Khan, Appl. Phys. Lett. 86, 021908 (2005).

${ }^{12}$ P. Corfdir, P. Lefebvre, J. Levrat, A. Dussaigne, J.-D. Ganière, D. Martin, J. Ristić, T. Zhu, N. Grandjean, and B. Deveaud-Plédran, J. Appl. Phys. 105, 043102 (2009).

${ }^{13}$ T. Gühne, Z. Bougrioua, P. Venneguès, M. Leroux, and M. Albrecht, J. Appl. Phys. 101, 113101 (2007).

${ }^{14} \mathrm{P}$. Vennéguès, Z. Bougrioua, and T. Guehne, Jpn. J. Appl. Phys., Part 1 46, 4089 (2007)

${ }^{15}$ M. Merano, S. Sonderegger, A. Crottini, S. Collin, P. Renucci, E. Pelucchi, A. Malko, M. H. Baier, E. Kapon, B. Deveaud, and J.-D. Ganière, Nature (London) 438, 479 (2005).

${ }^{16}$ J.-M. Bonard and J.-D. Ganière, J. Appl. Phys. 79, 6987 (1996).

${ }^{17}$ C. M. Parish and P. E. Russell, Appl. Phys. Lett. 89, 192108 (2006).

${ }^{18}$ J. Mickevičius, M. S. Shur, R. S. Qhalid Fareed, J. P. Zhang, R. Gaska, and G. Tamulaitis, Appl. Phys. Lett. 87, 241918 (2005).

${ }^{19} \mathrm{~A}$ discussion of these extensions is proposed in P. Corfdir, P. Lefebvre, J. Ristić, P. Valvin, E. Calleja, J.-D. Ganière, and B. Deveaud-Plédran, J. Appl. Phys. 105, 013113 (2009).

${ }^{20}$ P. P. Paskov, R. Schifano, B. Monemar, T. Paskova, S. Figge, and D. Hommel, J. Appl. Phys. 98, 093519 (2005). 